Article

\title{
An Analysis of Rhythmic Patterns with Unsupervised Learning
}

\author{
Matevž Pesek $^{1, *(\mathbb{D})}$, Aleš Leonardis ${ }^{1,2}$ and Matija Marolt ${ }^{1}$ \\ 1 Faculty of Computer and Information Science, University of Ljubljana, Vecna pot 113, 1000 Ljubljana, \\ Slovenia; a.leonardis@cs.bham.ac.uk (A.L.); matija.marolt@fri.uni-lj.si (M.M.) \\ 2 School of Computer Science, University of Birmingham, University Rd W, Birmingham B15 2TT, UK \\ * Correspondence: matevz.pesek@fri.uni-lj.si
}

Received: 18 October 2019; Accepted: 20 December 2019; Published: 25 December 2019

\begin{abstract}
This paper presents a model capable of learning the rhythmic characteristics of a music signal through unsupervised learning. The model learns a multi-layer hierarchy of rhythmic patterns ranging from simple structures on lower layers to more complex patterns on higher layers. The learned hierarchy is fully transparent, which enables observation and explanation of the structure of the learned patterns. The model employs tempo-invariant encoding of patterns and can thus learn and perform inference on tempo-varying and noisy input data. We demonstrate the model's capabilities of learning distinctive rhythmic structures of different music genres using unsupervised learning. To test its robustness, we show how the model can efficiently extract rhythmic structures in songs with changing time signatures and live recordings. Additionally, the model's time-complexity is empirically tested to show its usability for analysis-related applications.
\end{abstract}

Keywords: music information retrieval; rhythm analysis; compositional hierarchical model

\section{Introduction}

Musical rhythm represents one of the basic elements of music. Several different aspects of rhythm and its perception have been explored in the fields of psychology [1], musicology and music theory [2,3], and music information retrieval (MIR) [4]. Rhythm is directly related to tempo [5]; moreover, rhythm may affect and change the perception of tempo without changing the latter. Rhythmic patterns significantly affect both the melodic and harmonic aspects of a music piece. By changing the underlying rhythmic structure, two versions of a song may be classified into different music genres and imply different dancing styles.

Due to its importance, rhythm and its related concepts of tempo and beat have been extensively explored in the music information retrieval field. In the following subsections, we present a cross section of related works and then provide motivation for developing a new model, presented in this paper.

\subsection{Related Work}

In the field of music information retrieval, many rhythm, tempo, and beat related problems have been explored. According to Bello et al. [4], there are several established perspectives on musical rhythm. Four tasks are formalized within the Music Information Retrieval Evaluation eXchange (MIREX) (http://www.music-ir.org/mirex/wiki/MIREX_HOME) [6]: genre estimation, tempo estimation, beat tracking and downbeat estimation. 


\subsubsection{Established MIR Tasks}

The audio genre classification task is closely related to rhythm since rhythmic patterns represent one of the key features for differentiation between music genres. For example, Dixon et al. [7] tackled the problem of dance music genre classification by identifying different patterns, which define each music genre. They evaluated their approach on the Ballroom music dataset, which contains eight music genres: Jive, Cha cha, Quickstep, Rumba, Samba, Tango, Viennese Waltz and (English) Waltz. They showed that the rhythmic patterns are a useful feature for genre classification. Esparza et al. [8] proposed a neural network for rhythm genre classification and evaluated it on the Latin Music Dataset where their approach achieved state of the art results. They further explored the underlying rhythmic structures and pointed out several limitations in the dataset. Their research also showed that a single genre is not necessarily defined by a single specific rhythm in dance music due to inter-genre influence.

The tempo estimation task, as one of the first MIREX tasks, is also closely related to the rhythmic aspect of music. In recent years, deep learning has been used for tempo estimation. For example, Böck et al. [9] proposed an approach based on recurrent neural networks in combination with comb filters. As a generalization of tempo estimation, the goal of beat tracking is to identify the positions of beats in the audio. Although the task seems relatively trivial, current F-measures of the best approaches on different datasets still only reach around 0.6-0.7 (MIREX 2018) [10,11]; therefore, there is still room for improvement. The downbeat estimation task extends beat tracking by also identifying the first beat of each measure. To reduce the dominance of $3 / 4$ and $4 / 4$ meters prevalent in Western music, several non-Western music datasets (e.g., Turkish, Cretan and Carnatic) are used for evaluating the algorithms for this task. Due to the strong interrelationship of meter, beat, and tempo, several approaches attempt to model more than one aspect of rhythm. For example, Krebs et al. [12] proposed a Hidden Markov model-based system, which they applied to beat tracking and downbeat estimation. They also evaluated their system on the Ballroom dataset.

\subsubsection{Models for Music Understanding}

Next to MIREX-type tasks, several researchers explored modeling of musical rhythm, where the focus is on the model and understanding of the modeled structures. These models often rely on analyses of human cognitive processes, and the perception of music and language.

One of the more recently established and frequently used music theories was proposed by Huron [13,14], based on six perceptual principles. He empirically shows that many of the commonly known music rules have their basis in the perceptual principles. Inspired by Huron's work, Abdallah and Plumbley [15] proposed a simple Markov chain model for rhythm modeling, to explore interconnections between entropy rate and aesthetic value. They applied the proposed model to two pieces of minimalist music and showed that their rather simplistic model produces a similar analysis to one provided by a human expert. Sioros et al. [16] proposed a generative model for characterization of musical rhythms by using transformations, which provide a musically meaningful encoding of patterns, focusing on the syncopation in rhythmic patterns. The model incorporates Huron's metrical expectation principle of weak-strong bonds to model syncopation. The authors demonstrated the model's performance on a pop music piece by Michael Jackson. They argue the proposed model's explicitness and usability for analysis, by providing "musically and perceptually meaningful and detailed characterization" (p. 126). The proposed model extends beyond the analysis of rhythm with its generative nature.

In contrast to models based on human perception, data-driven models learn implicit rules by extracting knowledge from large datasets. For example, Foscarin et al. [17] proposed a model based on probabilistic context-free grammars trained on quantized MIDI files from music21 (http:/ / web.mit. edu/music21/doc/about/referenceCorpus.html) and EWLD [18] datasets. Starting with unweighted acyclic grammars, this approach builds minimal parse trees from the music input. By adding additional restrictions and limiting the amount of all possible rules to be learned from the input, the model produces meaningful representations of rhythm structure and rhythmic notation. 
Temperley [19] proposed a unified model for polyphonic music analysis, including metrical analysis. In this later work, Temperley [20] focused on the rhythmic modality and compared six probabilistic models of rhythm on two datasets, the Essen Folksong Collection, and the corpus of Mozart and Haydn string quartets, to explore ways of modelling rhythm perception. The author argues that the perception of common-practice rhythm is governed by general principles. There are several hypotheses discussed, each arguing one of the six models presented: the Uniform Position Model, the Zeroth-Order Duration Model, the Metrical Position Model, the Fine-Grained Position Model, the First-Order Metrical Duration Model and the Hierarchical Position Model. The latter is based on the hypothesis that "the common-practice rhythms are generated in a hierarchical manner" (p. 358). Among the six models, the Hierarchical Position Model and the First-Order Metrical Duration Model achieved the lowest cross-entropy, with the Hierarchical Position Model being severely less complex than the First-Order Metrical Duration Model.

Since rhythm in non-Western music contains a more extensive variety of different meters, several works are explicitly dedicated to exploration and rhythm modeling of non-Western music. Holzapfel [21] observed the rhythmic patterns (usul) in Turkish Makam music. He investigated how these patterns are related to note events and how the patterns are related to the meter as a latent mental construct. By investigating the rhythmic patterns in a large corpus of Turkish music, he proposed a methodology capable of identifying the differences between Western and Turkish music, and applied a maximal likelihood criterion for rhythm classification. Similarly, London et al. [22] explored African rhythmic patterns, focusing on three different music rhythms. In their study, they explore the complexity of the perceptual and cognitive relationships between rhythmic patterns and their interpretive metric frameworks. They also compared their data to the Turkish Makam music [21]. Panteli and Dixon [23] investigated the invariance of audio features for a description of rhythmic content of diverse music styles, including Afro-American, North-Indian, African, Latin-Brazilian and classical music styles.

\subsection{Motivation}

The aforementioned approaches range from theoretical models for music understanding, to automatic methods for classification and estimation of rhythmic events in audio and symbolic music representations. While the classification models often give adequate results for a specific task, they seldom focus on the perceptual and cognitive aspects of rhythm. Often, they are designed as black boxes (e.g., using neural networks), which are trained for the task, but have limited ability to investigate and explain the learned concepts. When trained through a supervised or unsupervised procedure, large datasets are needed, which are often difficult to obtain, especially when dealing with non-mainstream or non-Western music. On the other hand, the theoretical and cognitive models attempt to encapsulate the perceptual and cognitive processes but are more challenging to evaluate. Usually, a significant amount of music knowledge is built into these models by design, without a learning process. Therefore, while they may deliver feasible and human-understandable explanations of the underlying cognitive concepts, these approaches often operate as immutable systems, constrained by the embedded knowledge.

To bridge the gap between both types of approaches, we propose an unsupervised learning approach for modeling rhythm. The model is derived from the compositional hierarchical model (CHM) that was previously applied to tasks such as multiple fundamental frequency estimation [24] and pattern discovery from symbolic music representations [25]. The presented model-the compositional hierarchical model for rhythm analysis (RhythmCHM)—unsupervisedly learns a multi-layer hierarchy of rhythmic patterns ranging from simple structures on lower layers to more complex pattern compositions on higher layers. The learned hierarchy is fully transparent, which enables the structure of the learned patterns to be observed and explained. The model employs tempo-invariant encoding of patterns and can thus learn and perform inference on tempo-varying and 
noisy input data. It does not carry any predefined notions on the underlying musical meters and can perform on any type of (rhythmic) music.

Due to its transparency, the model can be used as an analytical tool for musicologists to aid the pattern discovery and analysis. Additionally, the output of the model-pattern occurrences, frequencies and their hierarchical structures-can be used as features for classification with machine learning [26]. In contrast to other theoretical or symbolic-input models, the proposed approach robustly models changes in rhythm, which are more common in non-popular music, and successfully models tempo variations in live music. The model can therefore aid the researchers in music-related fields, such as (ethno)musicology and music information retrieval.

The paper is structured as follows: in Section 2, we present the RhythmCHM focusing on its structure and incorporated mechanisms. In Section 3, we analyze the model's performance from several viewpoints: its ability to learn patterns which are unique to individual music genres, its performance on pieces with different meters and its robustness to varying tempo and variations present in live music. The model's scalability and time complexity are also explored. We conclude the paper in Section 5 with a discussion of the performed experiments and ideas for future work.

\section{The Compositional Hierarchical Model for Rhythm Modeling}

In this section, we present the compositional hierarchical model for rhythm modeling (RhythmCHM). The compositional hierarchical model for music information retrieval was first introduced by [27] and has been applied to several MIR tasks, including automated chord estimation and multiple fundamental frequency estimation [24], as well as finding repeated patterns and sections in symbolic music representations [25].

The compositional model is based on the idea that complex signals may be decomposed into simpler constitutive parts. These parts possess various levels of granularity and can be distributed across several layers, depending on their complexity. Starting from the first layer, which contains parts representing individual (atomic) events, successive layers are composed by combining (composing) parts from previous layers. The compositions are formed during learning by observing statistics of part occurrences and their relations. Since statistics is the driving force of learning, the compositional model can be learned in an unsupervised manner. The learned compositional hierarchy is transparent and enables inspection and analysis of the learned compositions either top-down or bottom-up, thus providing an explanation of the learned concepts. An abstract example of the compositional hierarchical model is shown in Figure 1.

The RhythmCHM model extracts a hierarchy of rhythmic patterns from its input. It is based on relative encoding of time in rhythmic structures, which is commonly used in rhythmic representations and is a necessity, as an individual rhythmic pattern may vary in duration due to tempo changes within a music piece or due to different tempi across pieces in a music corpus. The model also contains mechanisms that handle the variability of rhythmic patterns, which commonly occur during transitions between segments (e.g., drum transitions) and in segment repetitions (e.g., half-feel and double-feel).

The structure of the model is illustrated in Figure 2. In the following subsections, we present the model's features and implementation. 


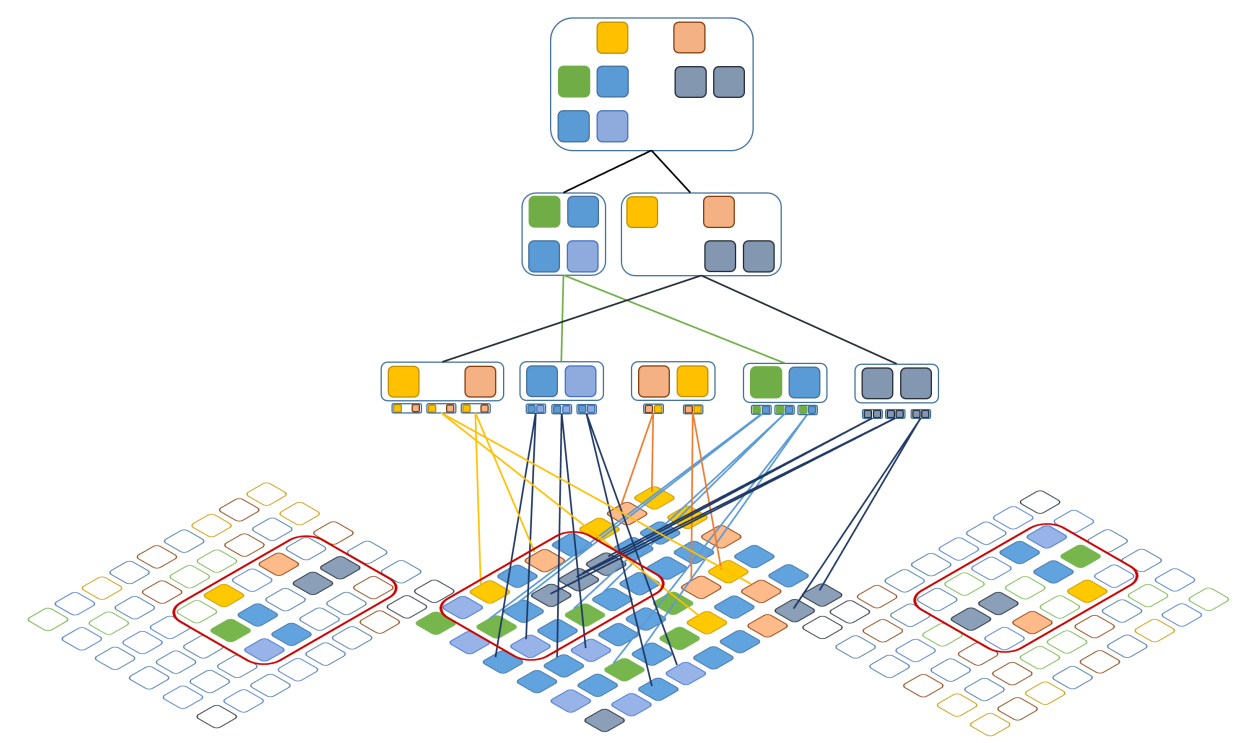

Figure 1. An abstract representation of the compositional hierarchical model. A composition of a complex structure in the input is marked in red. Each layer includes a set of parts which are compositions of parts from the previous layer. The structure may vary in its exact structure and position in the input. Part activations are depicted underneath each part on the first layer of compositions and represent occurrences of concepts in the input. Due to the relative encoding of parts, a part may activate at different locations within the input music signal. The entire structure of the model is transparent and allows for detailed analysis of the learned abstractions.

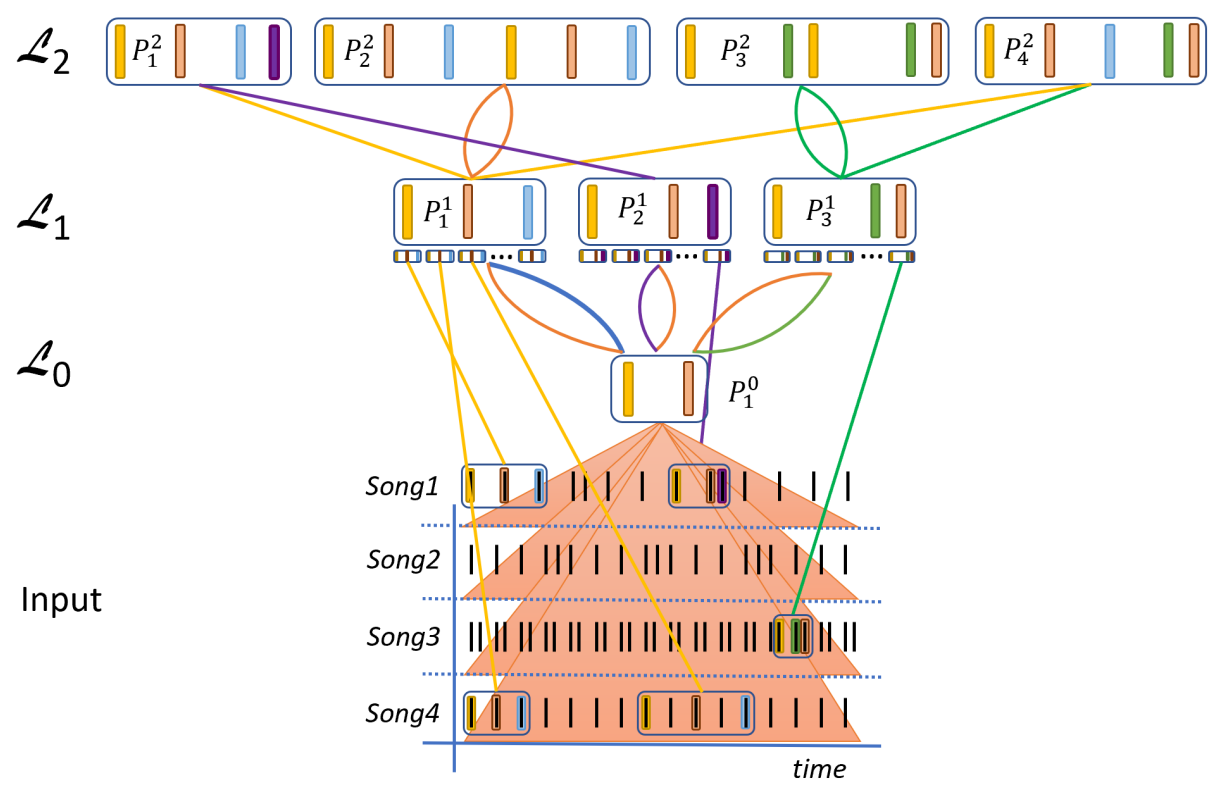

Figure 2. An abstract representation of a learned rhythmic hierarchy. Each layer consists of a set of parts which are compositions of parts from the previous layer. For each part, we display the rhythmic pattern it encodes, while the connections between parts show how compositions are formed. The compositions are formed of (sub)parts, where the second subpart is encoded relatively to the first part in the composition. The part on the first layer $P_{1}^{0}$ represents any occurrence of two input events. As the encoding of parts is time-invariant, parts activate wherever the patterns they encode occur in the input, regardless of their scaling. To illustrate this, we show three activations of the part $P_{1}^{1}$ with three different time scales in the input. Altogether, only five $\mathcal{L}_{1}$ activations are shown, others are omitted for clarity. 


\subsection{Model Description}

The input of the RhythmCHM model consists of onset times and magnitudes of music events. Pitches and note durations are ignored. Onsets may be extracted either from audio recordings (using an onset detector) or from symbolic representations. Formally, the input can be described as a set of onset time and magnitude tuples:

$$
\mathcal{I}:\left\{\mathbf{X}: \mathbf{X}=\left[N_{o}, N_{m}\right]\right\}
$$

The first layer of the model, denoted $\mathcal{L}_{0}$, consists of a single atomic part $P_{1}^{0}$, where the superscript is defined by the layer and the subscript marks the part number. Since any rhythm is composed of at least two events (i.e., a single event cannot solely represent a rhythmic pattern), the part $P_{1}^{0}$ represents any occurrence of two events (onsets) in the input signal. The relation between the two events is encoded in the part's activation, which is calculated when an input sequence is presented to the model.

The activation $A$ represents any occurrence of a part $P$ in the input and is composed of three elements: the onset time $A_{T}$ is the onset time of the first event, the magnitude $A_{M}$ is the activation magnitude, while the activation scale $A_{S}$ represents the scale of activation on the time axis. On the first layer, $A_{S}$ is defined as the difference of onset times of both events. Namely, as each part, corresponding to a rhythmic pattern in our model is relatively encoded, the activation scale represents the timing (tempo/duration) with which the pattern has been located in the model's input. The scale distinguishes between two pattern occurrences found at the same onset, one faster (small scale), and one slower (large scale). The activation magnitude $A_{M}$ represents the sum of event magnitudes. On the first layer, the magnitude represents the onsets' magnitudes $N_{m}$, as described in Equation (1). The magnitude is therefore always non-negative; however, it can be diminished or inhibited (i.e., zero value) due to the hallucination and inhibition mechanisms described later on in this Section.

More formally, the part $P_{1}^{0}$ activates for all pairs of input events $i_{1}=\left[N_{o}^{1}, N_{m}^{1}\right]$ and $i_{2}=\left[N_{o}^{2}, N_{m}^{2}\right]$, where $i_{1}$ occurs before $i_{2}$, as:

$$
A=\left\langle A_{T}, A_{S}, A_{M}\right\rangle \leftarrow\left\langle N_{o}^{1}, N_{o}^{2}-N_{o}^{1},\left(N_{m}^{2}+N_{m}^{1}\right) / 2 .\right\rangle
$$

\subsubsection{Rhythmic Compositions}

Each part on a successive layer $\mathcal{L}_{n}$ is a composition of one or more (sub-)parts on the previous layer $\mathcal{L}_{n-1}$. Formally, a composition $P_{i}^{n}$ of $K$ subparts $\left\{P_{k_{0}}^{n-1} \ldots P_{k_{K-1}}^{n-1}\right\}$ is defined as:

$$
P_{i}^{n}=\left\{P_{k_{0}}^{n-1},\left\{P_{k_{j}}^{n-1},\left(\mu_{1, j}, \sigma_{1, j}\right),\left(\mu_{2, j}, \sigma_{2, j}\right)\right\}_{j=1}^{K-1}\right\}
$$

Relations between the subparts forming the composition are governed by two Gaussians, which control their relative spacing and scaling, leading to a tempo invariant representation of a rhythmic pattern. All subparts relate to the first subpart of the composition.

The first Gaussian with parameters $\left(\mu_{1, j}, \sigma_{1, j}\right)$ defines the size of the subpart relative to the size of the first subpart. Values of $\mu_{1}$ larger than one indicate that the second subpart is longer than the first subpart; the values smaller than one indicate the inverse. When the model is trained on a corpus, the values of $\mu_{1, j}$ usually converge to integer ratios commonly present in different time signatures, e.g., $1 / 5,1 / 4,1 / 3,1 / 2,1,2$, etc. Given activations subparts $P_{k_{0}}^{n-1}$ and $P_{k_{1}}^{n-1}$, the value of $\mu_{1,1}$ is calculated as:

$$
\mu_{1,1}=\frac{A_{S}\left(P_{k_{1}}^{n-1}\right) *\left(1+\mu_{1}\left(P_{k_{1}}^{n-1}\right)\right)}{A_{S}\left(P_{k_{0}}^{n-1}\right) *\left(1+\mu_{1}\left(P_{k_{0}}^{n-1}\right)\right)} .
$$

The second Gaussian with parameters $\left(\mu_{2, j}, \sigma_{2, j}\right)$ defines the placement (onset) of the second subpart relative to the size of the first subpart. Thus, values larger than one indicate that the second subpart's onset comes after the end of the first subpart (there is a gap in between), the value of one 
means that the second subpart starts exactly at the end of the first subpart, while smaller values indicate overlap between both subparts. The parameter $\mu_{2}$ is calculated from activations of two subparts as:

$$
\mu_{2,1}=\frac{A_{T}\left(P_{k_{1}}^{n-1}\right)-A_{T}\left(P_{k_{0}}^{n-1}\right)}{A_{S}\left(P_{k_{0}}^{n-1}\right) *\left(1+\mu_{1}\left(P_{k_{0}}^{n-1}\right)\right)} .
$$

The parameter $\sigma_{1,1}$ is estimated from the distribution of pairs of activations with values near $\mu_{1,1}$, between $0.75-1.5$ times the first subpart activation scale. Similarly, $\sigma_{2,1}$ is estimated from the distributions of pairs of activations with values near $\mu_{2,1}$, within $0.75-1.5$ of the first subpart's length, therefore $\left[0.75^{*} \mu_{2,1}, 1.5^{*} \mu_{2,1}\right]$. These limits are imposed to exclude the influence of combinations of parts which could represent a double or half beat granulation, and would significantly skew the Gaussian.

In Figure 3, we show four simple first layer compositions with different values of $\mu_{1,1}$ and $\mu_{2,1}$. All parameters of a composition are learned during training and their values reflect the nature of the training data. For example, in studio-recorded music, tempo will be more even, so the variance of pattern lengths and their relative positions will be small, while the values will increase for live recordings.

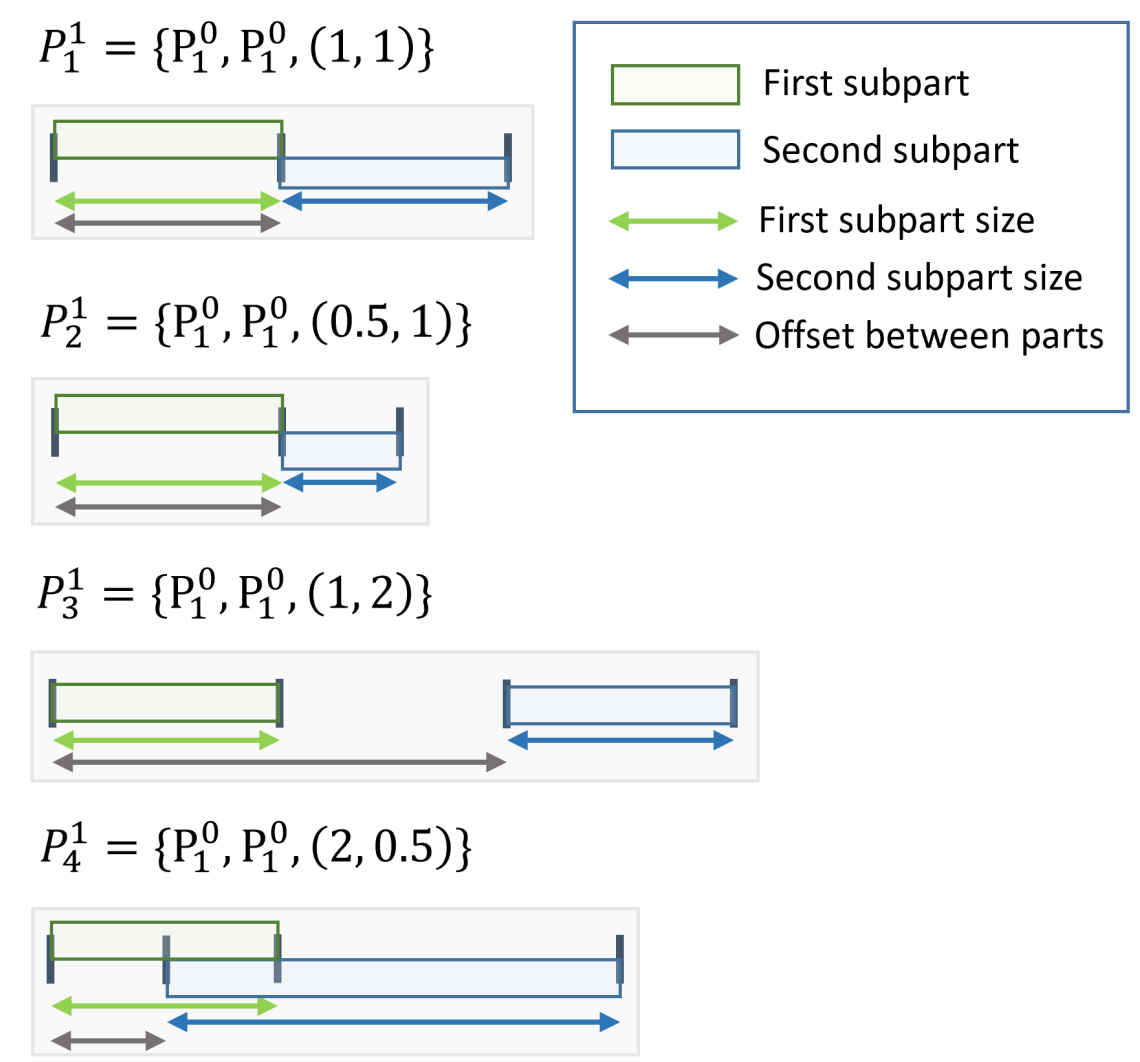

Figure 3. An example of four different RhythmCHM compositions. In the first example, the second subpart is the same length as the first subpart and is positioned exactly one $P_{1}^{0}$ length after the first subpart's position. In $P_{2}^{1}$, the second subpart is the same length as the first subpart and occurs at twice its length. In $P_{3}^{1}$, the second subpart is twice the length of the first subpart and starts at half its length. In $P_{4}^{1}$, the second subpart is half the length of the first subpart and starts exactly at its end. The green arrow represents the length (1) of the first subpart, while the blue arrow represents the length of the second subpart. The offset of the second subpart is marked with a gray arrow. 


\subsubsection{Activations of Parts on Higher Layers}

Activations of parts indicate the presence of the patterns they encode in the model's input. As already described, an activation has three components: time, which defines the starting time of the pattern; scale, which establishes the length of the (relatively encoded) pattern and magnitude, representing the activation's strength. A part can activate only if all of its subparts are activated with magnitude greater than zero (this constraint can be relaxed by the hallucination mechanism described later on). Due to the relative encoding of patterns in the model, a part can have many simultaneous activations (at different times and scales), indicating that the pattern it encodes has been found at several locations in the input signal.

An example is illustrated in Figure 4, which shows a simple $\mathcal{L}_{1}$ part with several activations at the same time but on different scales. Note that the part's encoding of a rhythmic pattern is relative and its scale is only established during inference on a concrete music piece. Thus, the model's parts encode rhythmic information independently of tempo, and in this way the model may easily follow patterns in pieces with changing tempo or in corpora that contain pieces in different tempi.

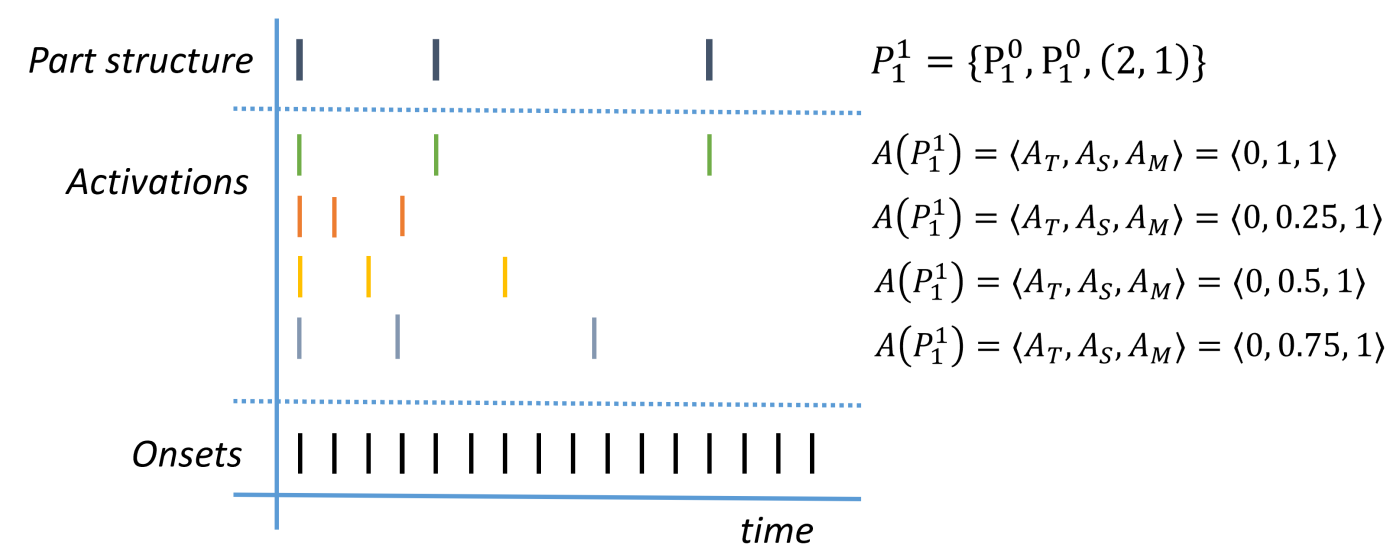

Figure 4. An $\mathcal{L}_{1}$ part with three events is activated on a simple input signal. Four activations are shown, all occurring at the same time, but with different scales encoded in $A_{S}$ (between 0.25 and 1).

\subsection{Learning and Inference}

The model is constructed layer-by-layer with unsupervised learning on a set of training examples, starting with $\mathcal{L}_{1}$. The learning process can be observed as a set-cover optimization problem, where we aim to find a minimal set of compositions for the learned layer, which would (through their activations) explain the maximal amount of information (events) present in the input data. The learning process is driven by statistics of part activations, which captures regularities in the input data.

\subsubsection{The Learning Algorithm}

We use a greedy approach to solve the set-cover problem. Learning takes place iteratively, where in each iteration, we pick a composition which covers the largest amount of uncovered events in the input and add it to the current layer. The learning algorithm is composed of two steps: finding a set of candidate compositions and adding compositions to the layer.

To construct the set of candidate compositions for a new layer $\mathcal{L}_{n}$, we first calculate activations of all parts from the layer $\mathcal{L}_{0}$ up to the layer $\mathcal{L}_{n-1}$. We then observe the co-occurrences of $\mathcal{L}_{n-1}$ part activations on the training set within a time window. The co-occurrences indicate parts, which frequently activate simultaneously in a certain relationship and are therefore candidates for forming compositions, as they are assumed to form common concepts. They are estimated from histograms of co-occurring part activations, which are formed according to the distances and scales of activation pairs. New candidate compositions are formed from parts where the number of co-occurrences 
exceeds the learning threshold $\tau_{L}$. The composition parameters $\mu_{1}, \mu_{2}$ and $\sigma_{1}, \sigma_{2}$ are estimated from the corresponding histograms (an example is given in Figure 5) and each new composition is added to the set of candidate compositions $\mathcal{C}$. The pseudo-code of the procedure is shown in Figure 6.

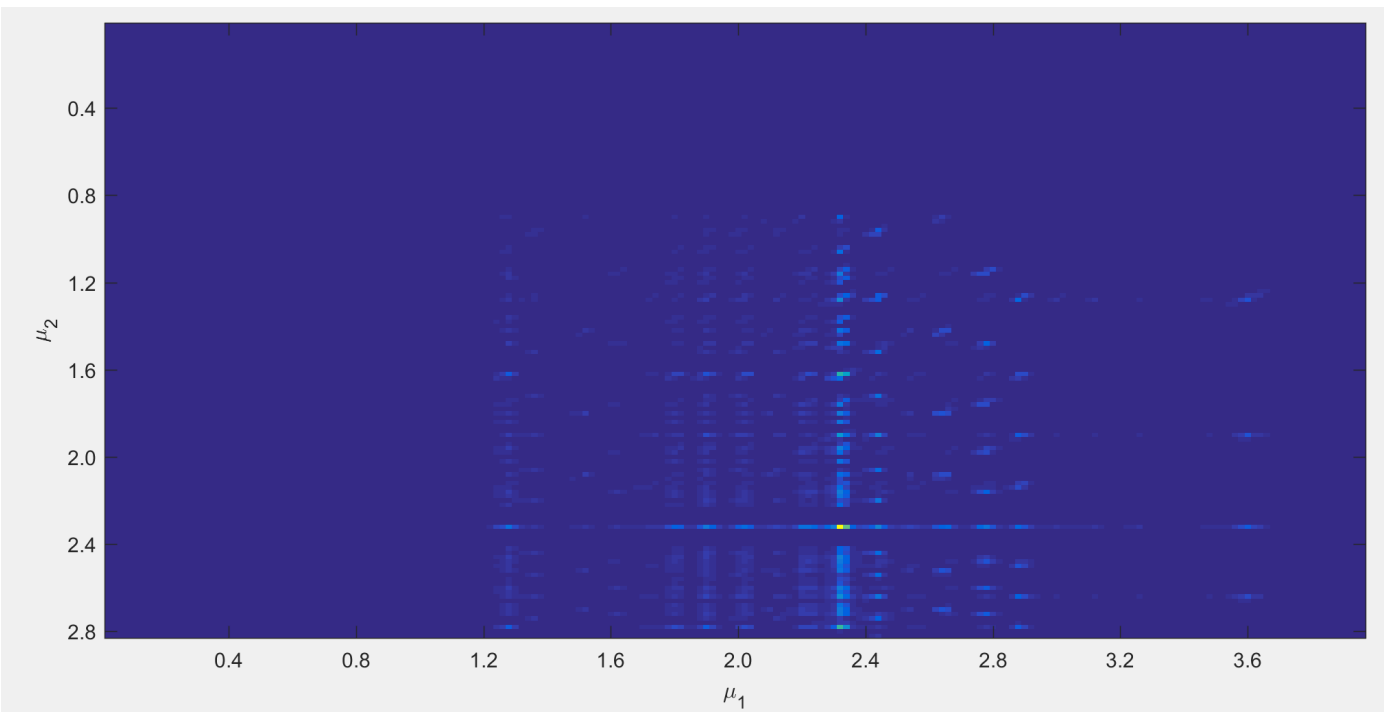

Figure 5. An example of the activation co-occurrence histogram. The axes represent the relative scale and locations of activations of a pair of parts.

1: procedure CANDIDATECOMPOSITIONS $\left(\mathcal{L}_{n}\right)$

2: $\mathcal{C}=\{\}$

3: for $\left(P_{1}, P_{2}\right) \in \mathcal{L}_{n-1} \times \mathcal{L}_{n-1}$ do

4: $\quad$ hist $\leftarrow \operatorname{array}(\max S i z e)$

5: $\quad$ for $A c t_{1} \in P_{1}$ do

6: $\quad$ for $A c t_{2} \in P_{2}$ do

7: $\quad$ if withinWindow $\left(\right.$ Act $_{1}$, Act $\left._{2}\right)$ then

8: $\quad \operatorname{loc} \leftarrow \operatorname{Act}_{2}\left[A_{T}\right]-\operatorname{Act}_{1}\left[A_{T}\right]$

9: $\quad$ scale $\leftarrow \operatorname{Act}_{2}\left[A_{S}\right] / \operatorname{Act}_{1}\left[A_{S}\right]$

10: $\quad$ hist $[$ loc, scale $] \leftarrow$ hist $[$ loc, scale $]+\operatorname{Act}_{1}\left[A_{M}\right]+\operatorname{Act}_{2}\left[A_{M}\right]$

11: $\quad$ end if

12: $\quad$ end for

13: end for

14: $\quad$ peak $\leftarrow$ peakPick $\left(\right.$ hist,$\left.\tau_{L}\right)$

15: $\quad$ while peak $\neq \varnothing$ do

16: $\quad[\mu, \sigma] \leftarrow$ estimateGaussian (hist, peak);

17: $\quad \mathcal{C} \leftarrow \mathcal{C} \cup$ newPart $\left(P_{1}, P_{2}, \mu_{1}, \sigma_{1}, \mu_{2}, \sigma_{2}\right)$

18: $\quad$ hist $\leftarrow$ removeFrom Hist (hist, peak, $\mu, \sigma)$

19: $\quad$ peak $\leftarrow$ peakPick $\left(\right.$ hist,$\left.\tau_{L}\right)$

20: end while

21: end for

22: return $\mathcal{C}$

Figure 6. The algorithm for generating histograms and candidate compositions.

From the set of candidate compositions $\mathcal{C}$, parts are iteratively chosen and added to the new layer as follows: 
- coverage of each part (events that part activations explain in the training set) is calculated,

- the part that adds most to the coverage of the entire training set is chosen. This ensures that only compositions that provide enough coverage of "new" data with regard to the currently selected set of parts will be added,

- the algorithm stops when the added coverage falls below the learning threshold $\tau_{C}$ or the overall coverage reaches the threshold $\tau_{P}$.

To avoid generating combinations, of which subpart activations are far apart, we limited the search with the withinWindow $\left(\right.$ Act $\left._{1}, A_{c t}\right)$ function. The pseudo-code for selecting the candidate compositions is outlined in Figure 7. Although the algorithm, which performs a greedy selection of compositions, does not provide the optimal subset of part candidates, its output is sufficient for the use in our model. The problem of candidate selection can be translated to a set-cover problem, which is known to be NP-hard. The greedy approach therefore represents an approximation of the best solution.

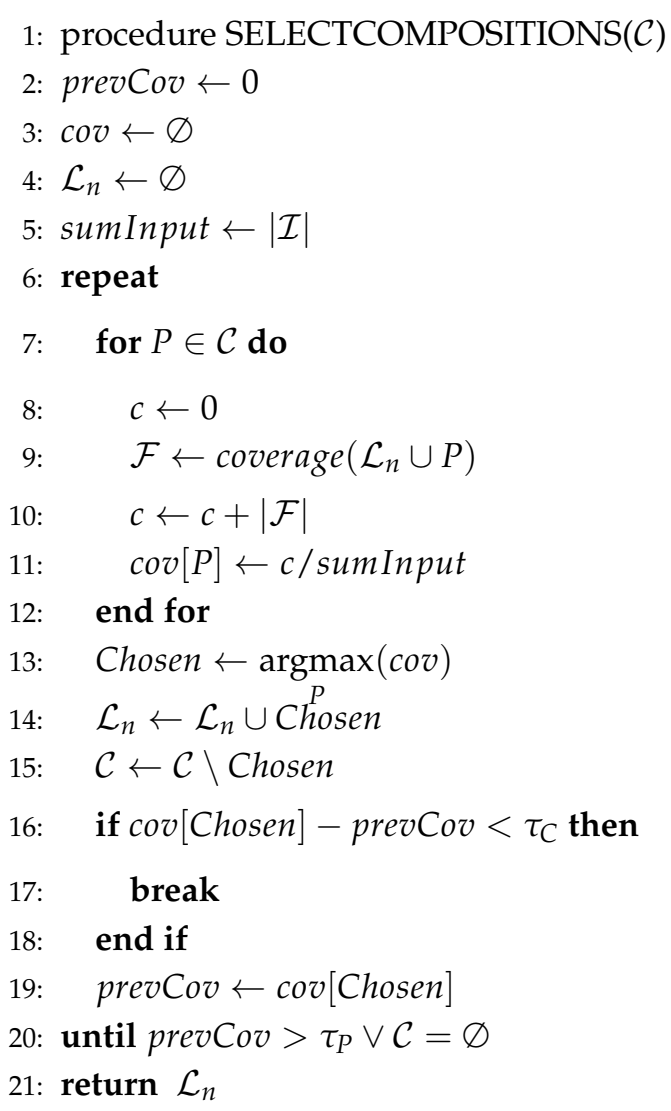

Figure 7. The greedy algorithm for selection of compositions from the candidate set $\mathcal{C}$. Compositions that add the most to the coverage of information in the learning set are prioritized.

The learning algorithm is repeated for each layer until a desired number of layers is reached. The desired number of layers is dependent on the complexity of the input and the specificity of the task. Simple rhythms can be observed on the first few layers, while higher layers contain more complex rhythmic structures that can be used for differentiating between different rhythms on a section-size level.

\subsubsection{Inference}

When a trained model is presented with new input data, the learned rhythmic patterns may be located in the input through the process of inference. Inference calculates part activations on the input data, bottom-up layer-by-layer, whereby the input data activates the layer $\mathcal{L}_{0}$. For each occurrence of 
a rhythmic pattern (part activation), the scaling factor, encoded in $A_{S}$, determines the length of the pattern, $A_{T}$ determines its onset time and $A_{M}$ its magnitude.

Inference may be exact or approximate. In the latter case, two mechanisms, hallucination and inhibition can be used to tolerate for rhythmic irregularities in music.

In exact inference, a part is activated only if all of its subparts are activated. Hallucination relaxes this condition and enables the model to produce activations even in the case of incomplete (missing, masked or damaged) input. The model generates activations of parts, which most fittingly cover the information present in the input signal, where fragments, which are not present, are hallucinated. The missing information is therefore extrapolated from the knowledge acquired during learning, encoded into the model structure. The amount of hallucination is governed by a threshold $\tau_{H}$.

Inhibition performs hypothesis refinement by reducing the number of part activations on individual layers. It provides a balancing factor in the model by reducing redundant activations. Although the learning algorithm penalizes parts redundantly covering the input signal, some redundant parts are retained. During inference, each layer may, therefore, produce multiple redundant activations covering the same information in the input signal. Inhibition removes activations of parts that have already been covered by stronger activations of other parts. The amount of removal can be controlled by the inhibition threshold $\tau_{I}$.

Approximate inference aids to the model's ability to find rhythmic patterns with deletions, changes or insertions, thus increasing its robustness. The inhibition mechanism has a more pronounced role in the rhythmic model, because of the relativity of the encoded patterns and, typically, high regularity of the input signal. Especially on the lower layers, a high number of activations representing simple straight rhythmic patterns emerges, typically out of a few simple parts activating at different scales and onsets. An example is given in Figure 8, which shows a series of activations of a simple $\mathcal{L}_{1}$ part on a regular input signal. For clarity, only two different scales are shown in this example.

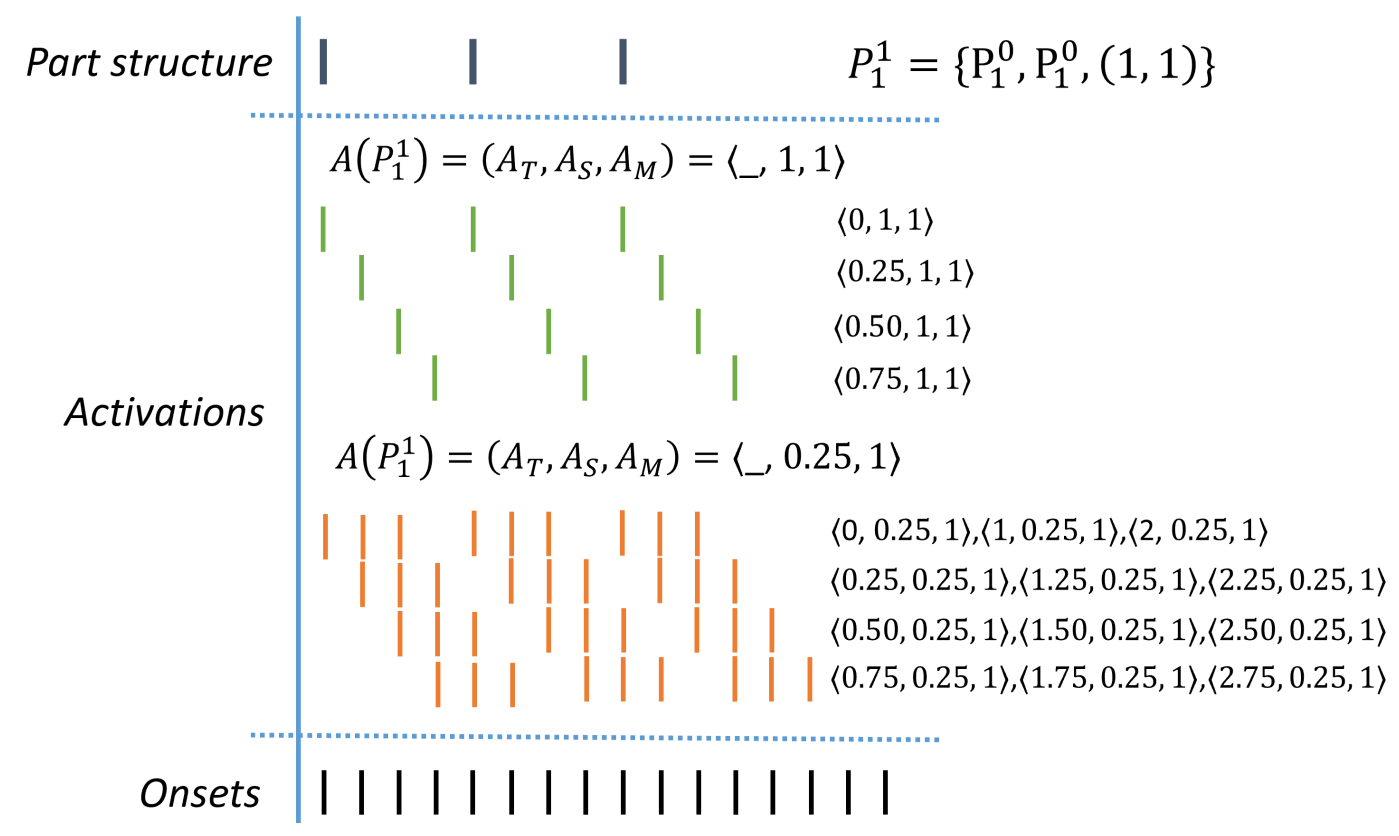

Figure 8. The example shows overlapping activations of a single $P_{1}^{1}$ part. Inhibition can be employed to efficiently reduce such overlapping activations. 


\section{Analyses}

We demonstrate the usability of the RhythmCHM model for extraction of rhythmic patterns in two experiments. In the first, we assess how the model can extract patterns from different dance music genres and how the extracted patterns characterize different genres. In the second, we show how the model can extract patterns from individual music pieces, in which rhythm changes due to changing time signatures, as well as tempo variations.

In both experiments, we extract rhythmic patterns from audio recordings. To detect the onsets of music events in audio, we use the CNN onset detector [28]. As the onset detector does not output onset magnitudes, we set all the magnitudes to the same value of one.

\subsection{Experiment 1: Analyzing Ballroom Dances}

We first evaluated the model's ability to extract rhythmic patterns from music corpora. For the purpose, we used the Ballroom dataset that is also used in many MIREX tasks (tempo and genre estimation, beat tracking). The Ballroom dataset [7] is publicly available online (http:/ / mtg.upf.edu/ ismir2004/contest/tempoContest/node5.html).

There are eight different genres in the Ballroom dataset-jive, rumba, cha cha, quickstep, samba, tango, Viennese waltz, and (English) waltz. We trained our model on all pieces for a subset of genres for which we expected to find distinctive rhythmic structures. For example, we expected to find swing patterns in jive and similar patterns in rumba and cha cha but in different tempi. In the following subsections, we report on the learned structures.

\subsubsection{Jive}

Jive music is based on a distinct swing rhythm, which contains unevenly spaced stressed events. In its everyday occurrence on radios, the swing rhythm is commonly associated with jazz. Jive is a medium to fast swing (commonly denoted as "uptempo") and usually contains a happy or goofy tune. Compared to other genres in the Ballroom dataset, jive is the only genre based on the swing rhythm.

In the model trained on all jive pieces in the dataset, we expected to observe distinct swing-like rhythmic structures. The learned model contained 38 parts on five layers (number of parts per layer: $\{2,8,10,9,9\}$ ). The analysis of the model revealed several compositions on different layers which contained the distinct swing rhythmic structure.

An example of such a structure on the fifth layer is given in Figure 9. More interestingly, as shown in Figure 10, the $\mathcal{L}_{5}$ part was generated from a single $\mathcal{L}_{4}$ part, the latter from a single $\mathcal{L}_{3}$ part and so on to the first layer. The first layer contains only two parts, where the first part $P_{1}^{1}$ represents three evenly separated events (straight rhythm), with $\mu_{1}=1, \mu_{2}=1$ and the second subpart $P_{2}^{1}$ an uneven rhythm with $\mu_{1}=0.86, \mu_{2}=1$. This part composes all the parts with swing rhythm structures on the higher layers $\mathcal{L}_{2} \ldots \mathcal{L}_{5}$.

Along with these structures, the model also learned parts representing the basic straight rhythm. The analysis of their activations showed that these parts activate on the downbeats of the rhythm, interpreting the input as a straight meter and ignoring the syncopated second beat. In addition, such parts also activated on the syncopated beats. This second group of activations, therefore, acted as straight meter with shifted phase. An example is given in Figure 11. 


\section{II II II II

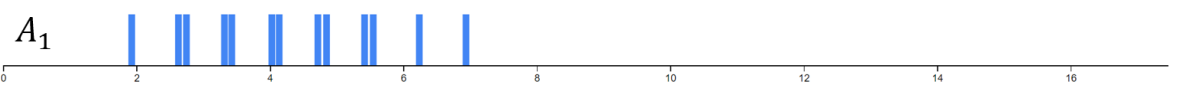

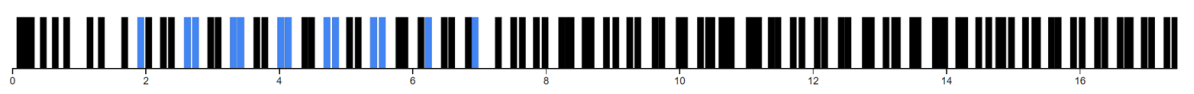

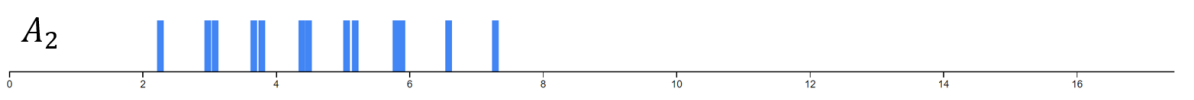

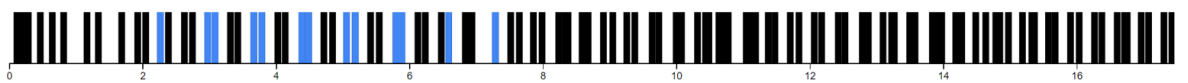

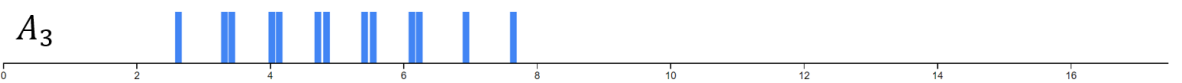

\section{|||||||||||||||||||||||||||||||||||||||||||||||||||||||||||||||||||||||||||||||||||||-||-}

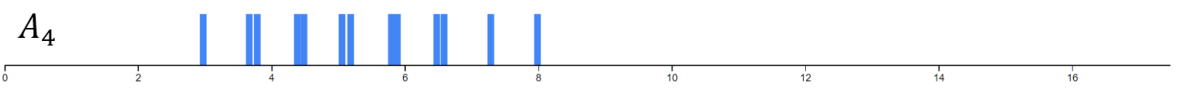

\section{||||||||||||||||||||||||||||||||| ||||||||||||||||||||||||||||||||||||||||||||||||||||-||}

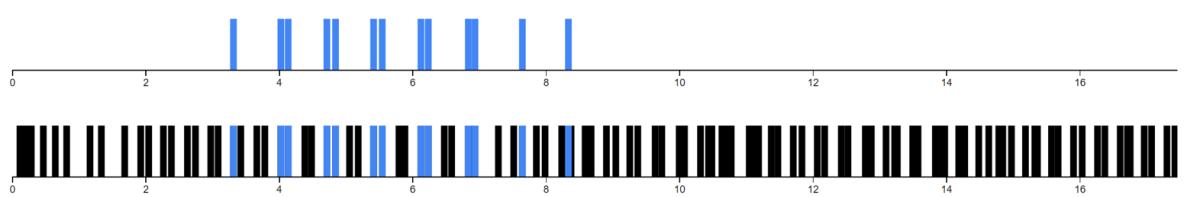

Figure 9. The $\mathcal{L}_{5}$ part representing the basic swing rhythm commonly found in jive songs. Below the part's structure, the first four activations are depicted individually with their projections onto the input layer. The decomposition of the part's structure is shown in Figure 10.

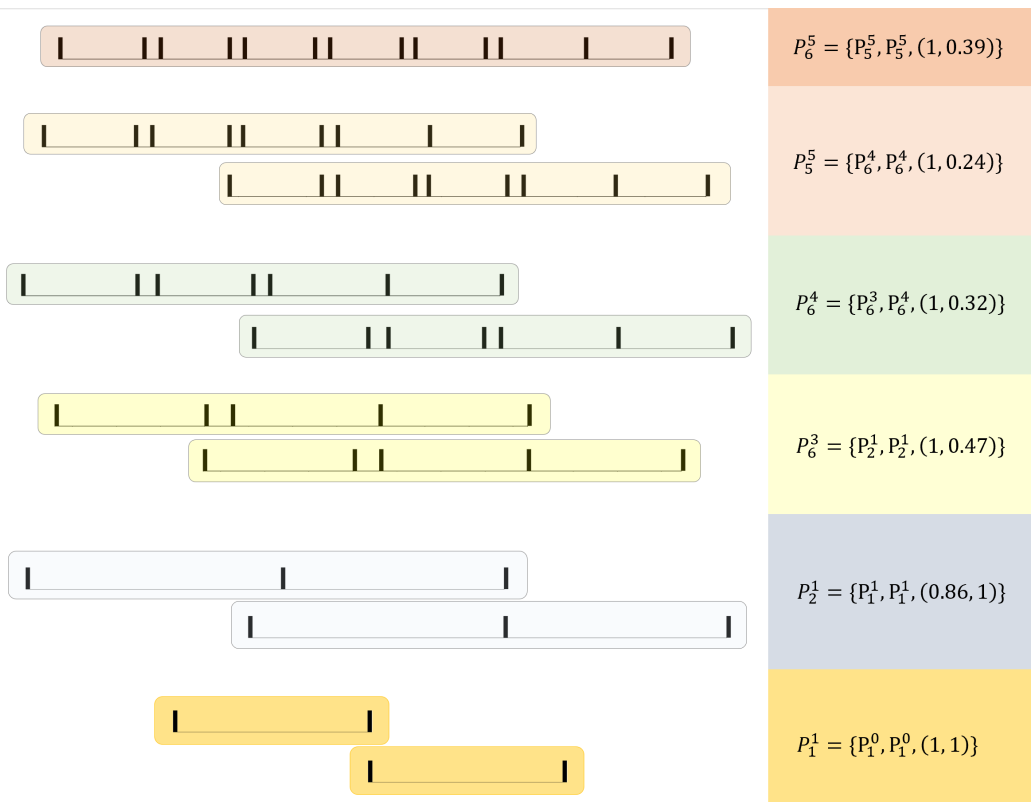

Figure 10. The structure of part $P_{5}^{6}$. All subpart compositions were formed from two instances of a single part on layers $\left\{\mathcal{L}_{0} \ldots \mathcal{L}_{5}\right\}$. The compositions' parameters are shown on the right side. Each part is shown twice with the offset used in the consecutive layer's composition. 

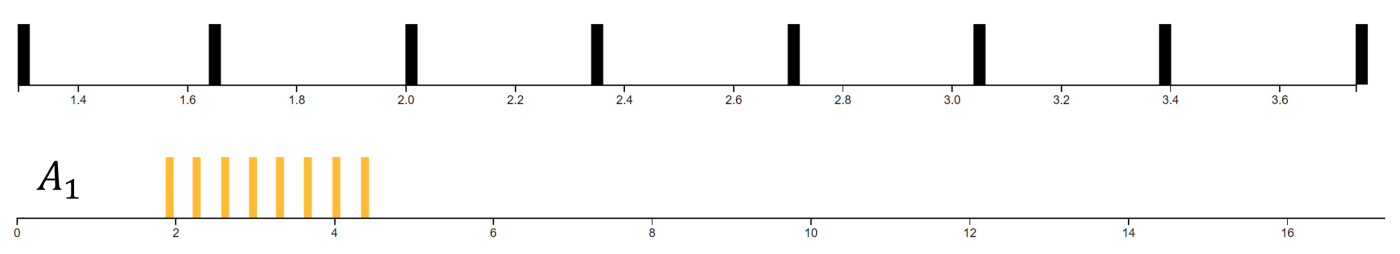

\section{|||||||||||||||||||||||||||||||||||||||||||||||||||||||||||||||||||||||}

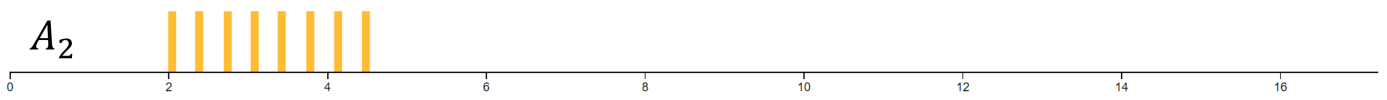

\section{||||||||||||||||||||||||||||||||||||||||||||||||||||||||||||||||||||||||||}

Figure 11. The $\mathcal{L}_{5}$ part representing a straight rhythm. Below the part's structure, two typical activations are depicted individually with their projections onto the input layer. The first activation covers eight consecutive downbeats, whereas the second activation covers the syncopation of the swing rhythm in the input, imitating straight rhythm with shifted phase.

\subsubsection{Samba}

Samba's rhythm partially resembles jive, with a distinctive difference in the timing of the second beat, which is played in straight rhythm. Due to its syncopation of the second beat, we can expect distinctive structures in the learned model, which cover the specific offset of the second beat in the rhythmic pattern. As shown in Figure 12, the model trained on samba songs from the dataset successfully captured the samba's basic beat structure. The structure itself may seem similar to jive; however, it differs in the ratio between the first and second beat. Compared to jive, the difference in ratios is about 15 percent.
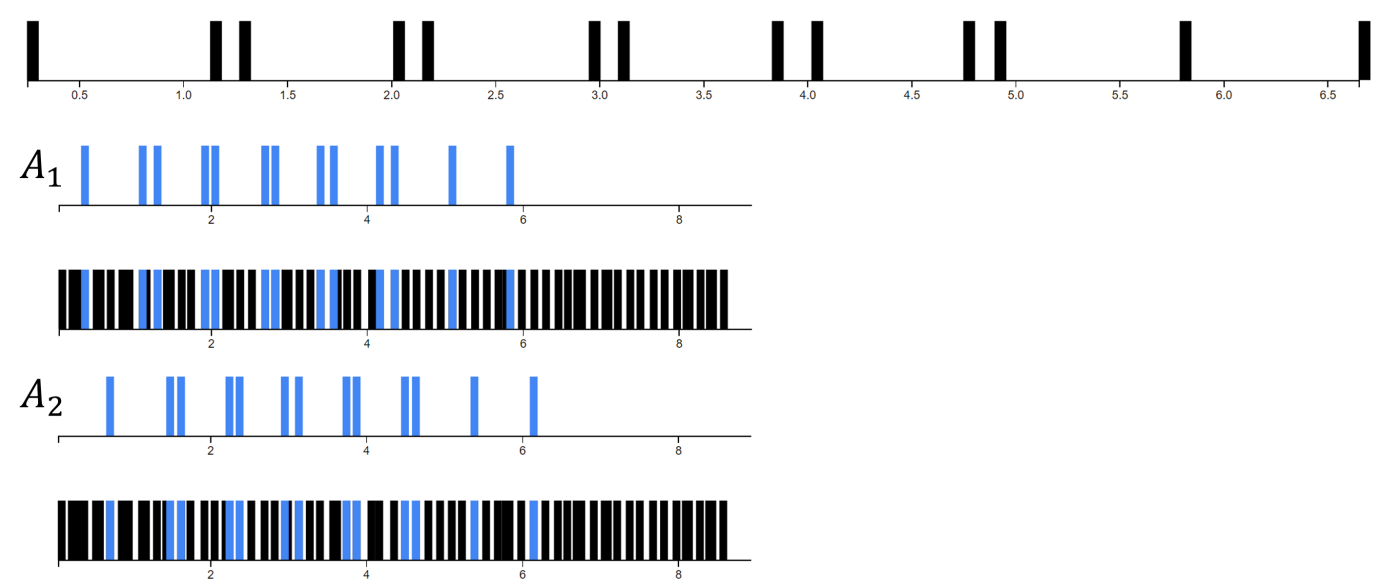

Figure 12. The $\mathcal{L}_{5}$ part representing the rhythmic pattern of a samba song. Below the part's structure, the first two activations are depicted individually with their projections onto the input layer. The part's structure is similar to jive, but contains different ratios between the first and the second beat.

\subsubsection{Rumba and Cha Cha}

Both music genres belong to the group of Latin-American dances. While the rumba dance and music are associated with sensual topics, the cha cha contains more bright, powerful and uptempo beats. Both music genres contain a distinctive "four-and-one" syncopation, which also defines the basic steps in the dance. Additionally, both genres are played in straight meter with strong accents on all four beats. 
We built a separate model for each genre and analyzed the learned structures. In contrast to the previously analyzed genres, these two models shared a greater deal of straight rhythmic parts. The rumba-trained model had 36 parts on five layers, while the cha-cha-trained model contained 33 parts.

Straight patterns dominated in both models. The distinctive patterns, containing the "four-and-one" beats, were not as dominant as we initially expected. This was mainly the effect of the variety of percussive and brass instruments playing granulated rhythmic riffs on and between all beats, which the onset detector does not distinguish from the others. Therefore, straight patterns dominate. The most closely associated typical pattern that was found is depicted in Figure 13.

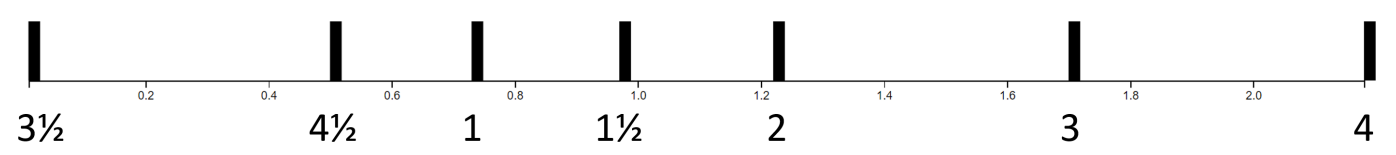

Figure 13. The most prototypical part found in the cha cha trained model on layer $\mathcal{L}_{3}$. The "four-and-one" pattern is partially explained. However, two other eight-note events are also included.

\subsubsection{Tango}

Although tango possesses South American roots, the widely known international (European) version of the dance contains several aspects influenced by the European culture, such as the closed position of the dancers, more common in standard dances. The music associated with this dance is usually orchestral, played with severe stress on the syncopation (usually played by the violin and other string instruments) on the offbeat before the downbeat.

In the trained model, we observed several parts forming a structure similar to the one displayed in Figure 14. The off-beat is usually located between two beats and creates a syncopated rhythm, for example, the first and the second beat in the meter, and can also appear on other locations. Onsets of these beats have a fast attack and decay and usually a higher magnitude. The beat after is usually omitted to create a deeper sensation of the stressed beats. Even though these properties are not reflected in the input, which only contains the onset times, the learned structures do reflect the specifics of the rhythm.

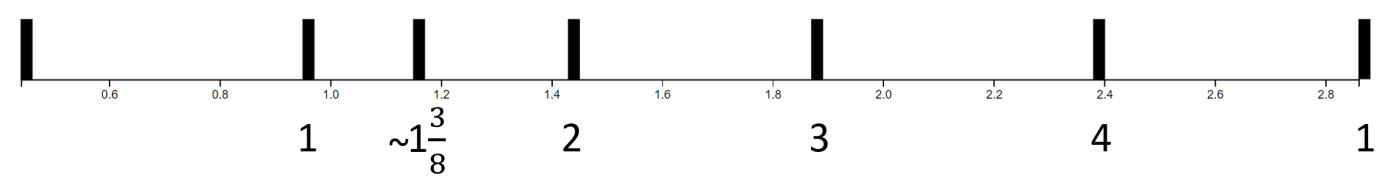

Figure 14. A tango part representing the regular "1-2-3-4" beats that match the meter and a syncopated off-beat (third onset from the left).

\subsection{Experiment 2: Robustness of the Model to Timing and Tempo Variations}

The compositional structures in the model encode individual rhythmic patterns in a relative representation. Such relative encoding makes the learned patterns independent of the underlying tempo and minimizes the amount of parts per layer. During inference, activations of these structures map them into absolute temporal space by scaling, where the scaling factor is encoded in the activations.

To demonstrate the model's ability to learn patterns regardless of the underlying tempo and its ability to encode tempo changes in the scaling factor of each activation, we learned a model on a Sirtaki song (Song available on Youtube: https://youtu.be/CbmbSjeUrxc, alternative version available here: https:/ /www.youtube.com/watch?v=dzlcxN0lxSo), which is well known for its rhythmic and tempo changes. 
Due to the relative encoding of patterns, the entire hierarchy contains few parts on its five layers- $\{2,3,7,8,8\}$. We show that by observing the scaling factor of part activations, we can infer tempo changes in the analyzed song. More specifically, we focus on the segment (60-150 s) during which the rhythm remains similar, but the tempo increases drastically. The segment starts with a tempo of about 90 beats per minute and gradually increases up to 210 beats per minute. In Figure 15 we show how the scale of activations of a $\mathcal{L}_{6}$ part, representing ten straight beats, changes. The scaling factor starts at 0.7 and decreases down to about 0.3 . The ratio between the initial and the final scale corresponds to the ratio between the initial and final tempo.

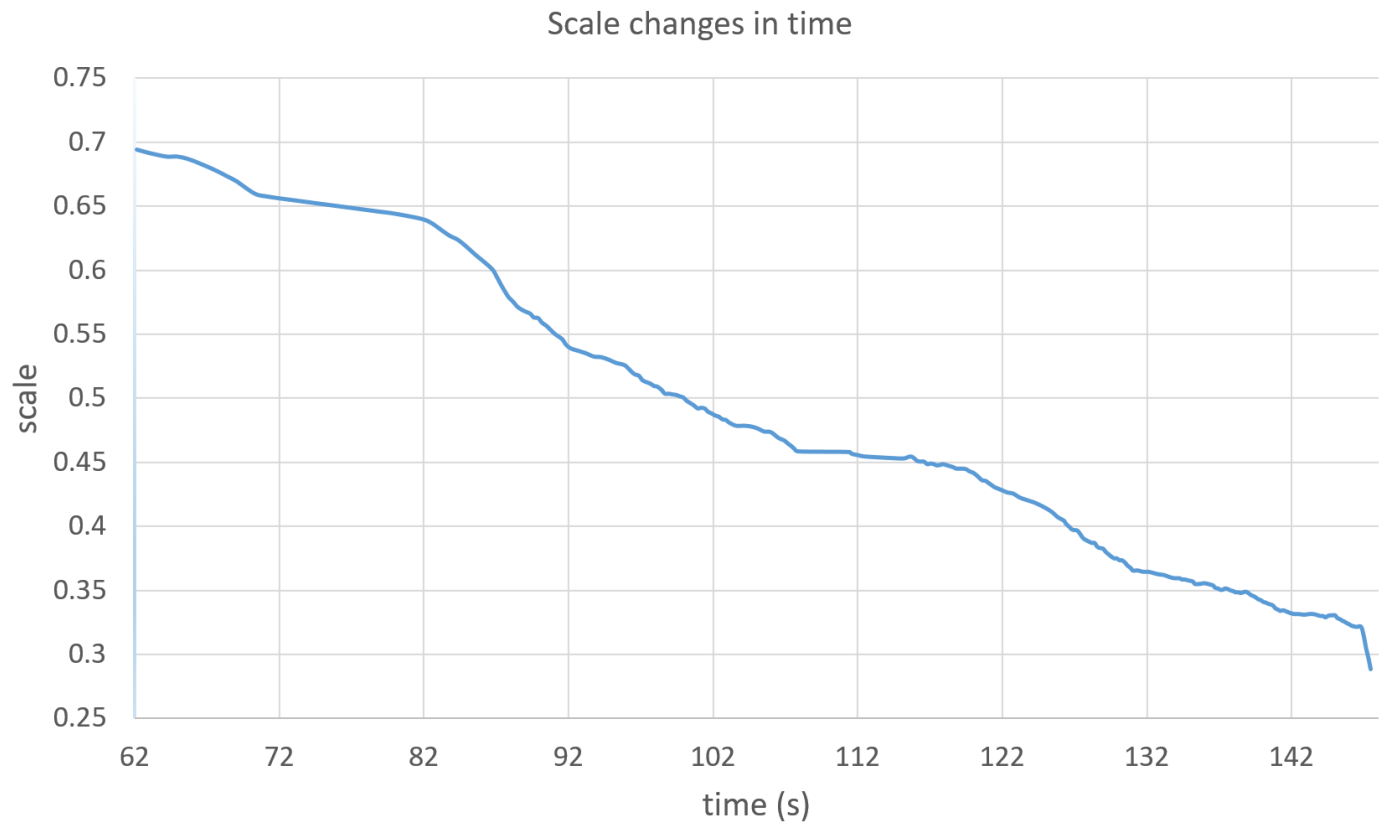

Figure 15. Scaling factor changes in the Sirtaki example as a consequence of tempo changes. The tempo changes from roughly 90 to 210 beats per minute, causing the scale of part on layer $\mathcal{L}_{6}$ to change from 0.7 to 0.3 .

To show the model's ability to robustly extract rhythmic patterns in different time signatures from a single music piece, we looked at live music recordings on Youtube. In this context, an interesting live recording was produced by the Croatian singer Severina in a song "Djevojka sa sela" (Song available on Youtube https:/ / youtu.be/heJQAckM-eI). The live version performed at her concert in 2009 was accompanied by trumpeters who played a $7 / 8$ meter in verse, whereas the rock band played a 4/4 meter in the refrain. By training the model on this song, we produced a 5 layer hierarchy, containing $\{3,11,11,10,9\}$ parts on layers $\left\{\mathcal{L}_{1} \ldots \mathcal{L}_{5}\right\}$, respectively. We first observed the three parts forming the first layer. The part structures are depicted in Figure 16.

As is evident in Figure 16, two parts $P_{1}^{1}$ and $P_{3}^{1}$ activate across the entire song, while one part activates only on the segments played in $7 / 8$ meter. On layer $\mathcal{L}_{2}$, we can find five parts which cover 5-6 rhythmic events, explaining the $7 / 8$ segments-as the $7 / 8$ meter can be broken into $3 / 8+2 / 8$ $+2 / 8$ structure, these parts successfully find the downbeats of all three submeters. The remaining six parts are the compositions of $\mathcal{L}_{1}$ parts, which cover both meters. Unfortunately, these parts also compose the majority of $\mathcal{L}_{3}$ parts and further. This is a consequence of the statistical nature of the model's learning. Namely, the parts covering both meters cover a greater portion of the events when compared to the parts covering only the $7 / 8$ meter segments. Therefore, the greedy learning procedure favours such parts on the higher layers and tends to ignore the $7 / 8$-meter candidates with smaller coverage. Nevertheless, the model successfully shows the ability to distinguish rhythmic structures with different meters on $\mathcal{L}_{1}$ and $\mathcal{L}_{2}$ layers. 
Moreover, we selected this particular song, as it has a very uneven tempo due to the live performance and the style of trumpet playing. The variations are visible in Figure 17, which shows variations in the scale factor of activations of a $\mathcal{L}_{2}$ part, implying large differences in the timing of the events covered by this part. The relative encoding of the compositions enables the model to extract the rhythmic structures robustly in such circumstances.

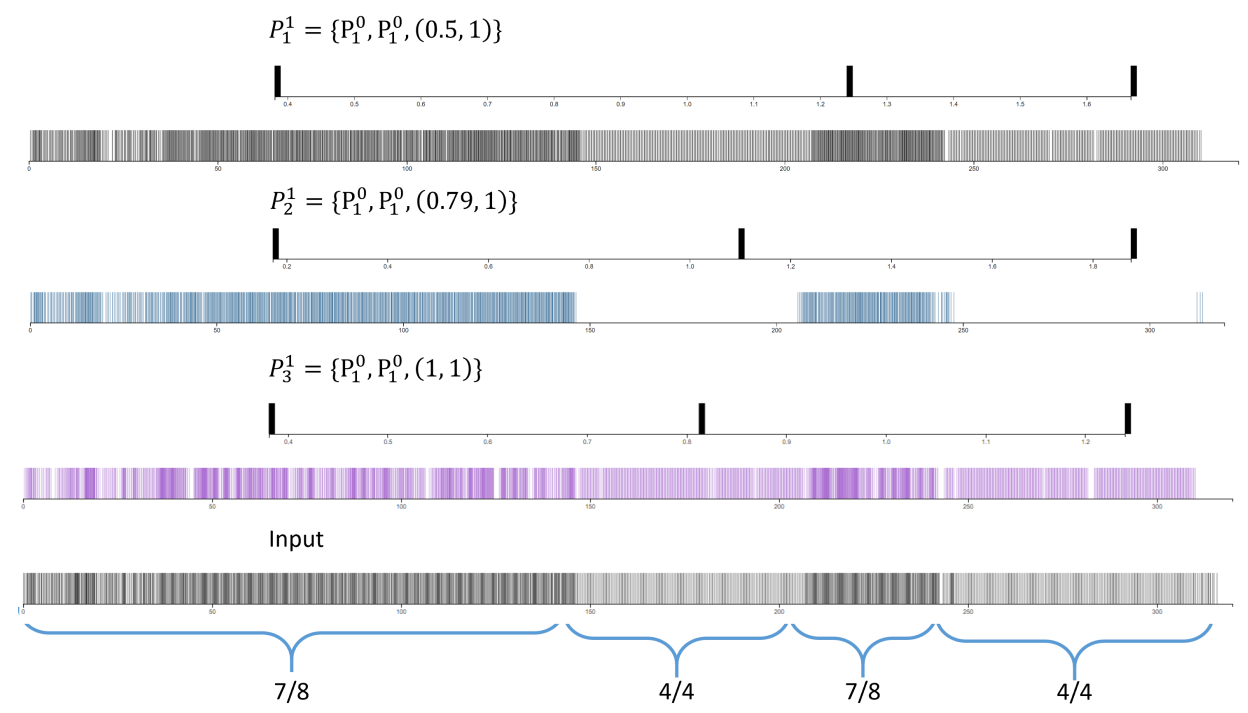

Figure 16. The three parts forming the $\mathcal{L}_{1}$ layer for the Severina's song. All activations for the parts $P_{1}^{1}$ and $P_{2}^{1}$ are shown in violet and blue colors respectively. It may be clearly observed that the $P_{2}^{1}$ part covers the input song segments played in $7 / 8$ meter, while $P_{1}^{1}$ and $P_{3}^{1}$ activate across both $7 / 8$ and 4/4 meters segments.

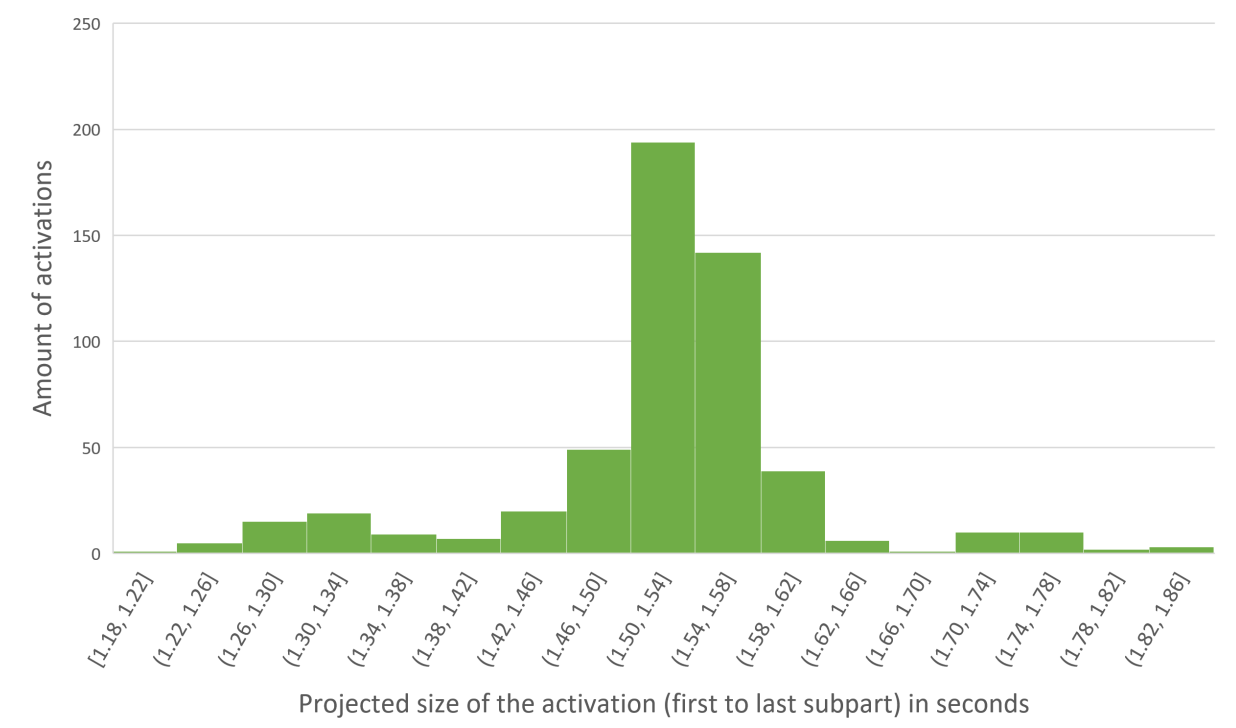

Figure 17. Varying activation sizes for a layer 2 part which activates on the $7 / 8$ meter segments. The majority of activations is within 100 milliseconds of the average size of 1.52 seconds (about $6 \%$ difference in tempo); however, many occurrences also range from about $25 \%$ slower up to $25 \%$ faster).

\section{Scalability and Visualization}

\subsection{Scalability}

The goal of the presented compositional model is to provide an automated tool for rhythm analysis of individual songs or entire corpora. Thus, scalability also plays an important role in its 
applicability. The users should be able to use the model on their computers and mobile devices without specialized hardware and large amounts of working memory. In this section, we first assess the time complexity of model training, which is the most time-consuming part of model usage.

In order to evaluate how the model scales with the amount of training data, we trained 11 five-layer models on datasets ranging from 400 to 350,000 events (onsets). The training dataset consisted of files from the ballroom dataset, augmented with 30-second clips of songs in a variety of popular music styles.

The results are shown in Table 1 . The time needed to train a model grows linearly with the number of events. Training of a five-layer compositional structure on a database of approx. 350,000 events take 50 min on a single core CPU. The linear dependency is clearly visible in Figure 18. Although a single CPU core was used in our experiments, the model implementation is parallelized in most stages: during learning, the generation of histograms, as well as candidate picking are both parallelized. The inference process is also parallelized, except for the inhibition mechanism.

Table 1. The table summarizes the time needed and the number of learned parts when learning a five-layer model with a different number of input events. The left side of the table shows the number of music files and events in the input, along with the time needed to train a five-layer hierarchy. On the right side, the number of parts per individual layer, along with the sum of all parts in the hierarchy, is displayed.

\begin{tabular}{rrrllllll}
\hline \# of Files & Time (s) & Events & $\mathcal{L}_{\mathbf{1}}$ & $\mathcal{L}_{\mathbf{2}}$ & $\mathcal{L}_{\mathbf{3}}$ & $\mathcal{L}_{\mathbf{4}}$ & $\mathcal{L}_{\mathbf{5}}$ & \# of Parts \\
\hline 2 & 3.77 & 389 & 2 & 6 & 9 & 10 & 9 & 36 \\
4 & 5.16 & 660 & 3 & 10 & 9 & 9 & 10 & 41 \\
8 & 10.43 & 1175 & 5 & 10 & 9 & 9 & 10 & 43 \\
16 & 9.16 & 2307 & 3 & 10 & 9 & 8 & 9 & 39 \\
32 & 20.13 & 4754 & 3 & 9 & 9 & 8 & 8 & 37 \\
64 & 86.98 & 11,097 & 4 & 9 & 10 & 8 & 9 & 40 \\
128 & 171.00 & 22,892 & 4 & 9 & 9 & 8 & 8 & 38 \\
256 & 382.47 & 45,229 & 4 & 10 & 9 & 9 & 9 & 41 \\
512 & 704.29 & 86,118 & 4 & 10 & 10 & 8 & 8 & 40 \\
1024 & 1587.78 & 171,585 & 4 & 10 & 10 & 9 & 9 & 42 \\
2048 & 3092.72 & 347,863 & 4 & 10 & 10 & 10 & 10 & 44 \\
\hline
\end{tabular}

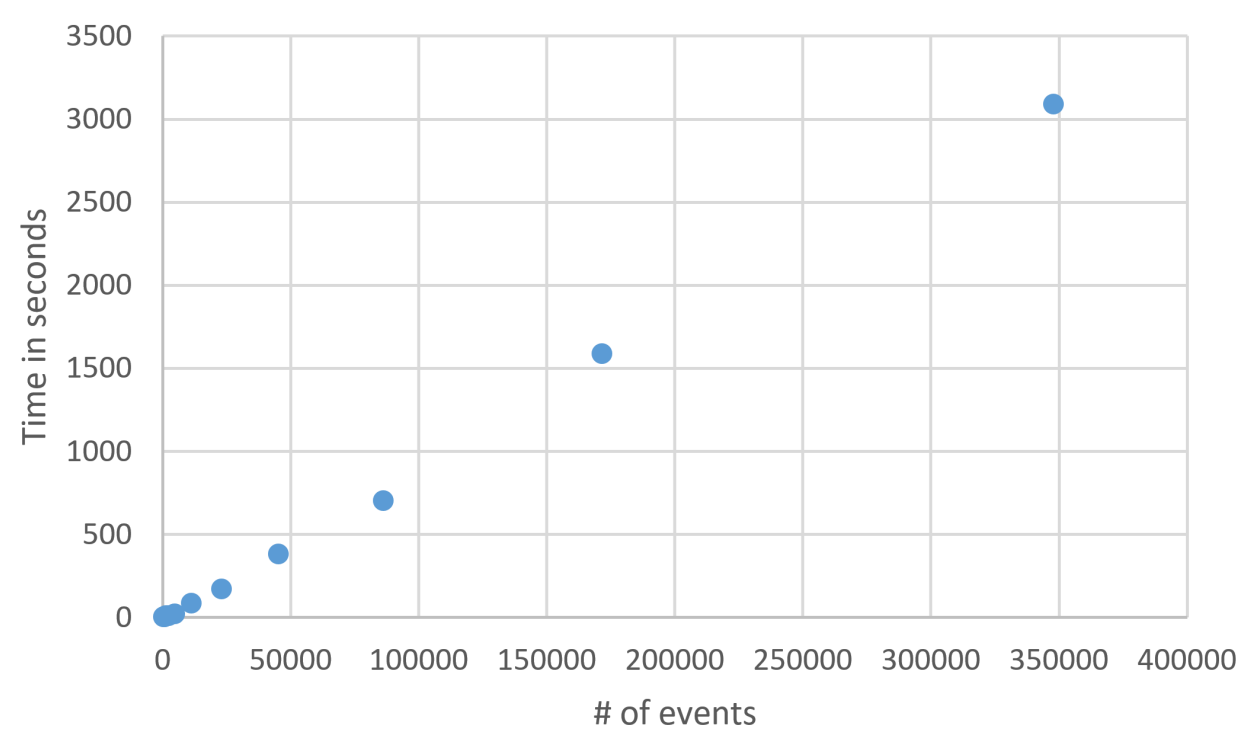

Figure 18. The graph shows the time needed to train a model (in seconds) in relation to the number of input events. The dependency is linear. 
The amount of parts per layer grows only slowly with larger training datasets. Due to the relative encoding of the learned structures, even small datasets can already produce parts general enough to cover a variety of input data. The first layer is always small (3-4 parts), while the number of parts on higher layers remains approximately constant (8-10 parts). Next to relative encoding, this is also likely due to the similar rhythmic structures found in the dataset-it mostly covers popular music genres. Using a more varied training dataset (e.g., music with very different metric structure) would likely increase the number of parts. The results of this experiment show the model's usability for rhythmic analysis of large music corpora.

\subsection{Visualizing the Patterns}

Due to its transparency, the model can be utilized for visualization and exploration of the learned hierarchy of rhythmic patterns and their occurrences within training data, as well as in new and unseen data, within individual songs, or entire corpora. For this purpose, we developed a web visualization tool to bring the model closer to the interested researchers.

Our visualization enables simple one-click inspection of individual model parts and occurrences of rhythmic patterns they encode. An example is shown in Figure 19. Each part (pattern) is displayed in a different color. Its description provides information on the part's structure, as well as the total coverage and activation magnitudes on the input data. When a pattern is selected, its structure is also visualized (a straight pattern of nine events is shown in the Figure). All pattern occurrences are shown as a list, as well as projected onto the input set of events at the bottom of the visualization. Based on their goal, users may select individual activations or observe all activations of a specific pattern. The visualized activations and the input are visualized at the bottom of the interface. The web interface is publicly accessible at http:/ /rhychm.musiclab.si/.

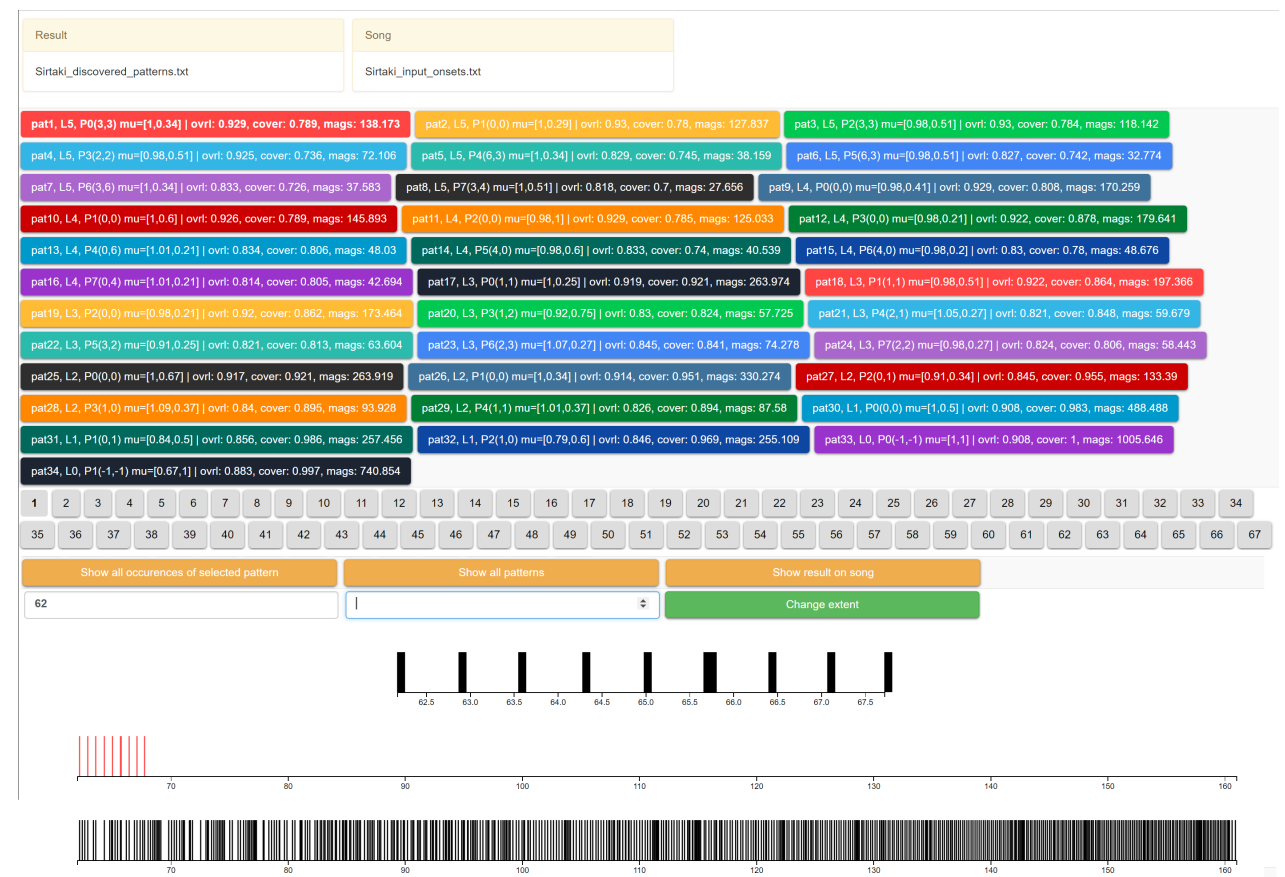

Figure 19. The web-based visualization of a model, showing a straight pattern and its occurrences in the input. Each part (assigned an individual color) is described by the composition of subparts and their offsets $\mu_{1}, \mu_{2}$, with some additional information, such as coverage of the input signal, self-overlapping of the activations and the amount of covered events in the input signal. Each individual part can be inspected by visualizing its structure and its projected activations onto the input visualization. The time-span (in seconds) of the projection can be narrowed to the segments of interest. 


\section{Conclusions}

We presented a novel compositional hierarchical model for rhythm analysis. Its features are unsupervised learning of relative tempo-invariant rhythmic structures, transparency, which enables inspection of the learned concepts, and robustness in learning and inference.

We applied the model to recordings in the Ballroom dataset to analyze whether genre-specific rhythmic structures can be learned. A separate model was trained for each genre. Due to transparency, the learned structures were clearly interpretable and their analysis showed that the model is capable of extracting rhythmic structures specific to individual genres. Additionally, we showed on different examples that the model can robustly learn and infer patterns in pieces with changing tempo and timing variations, as well as extract patterns corresponding to different time signatures. The model can be unsupervisedly trained on a single music piece or on a corpus. A model trained and inferred on a dataset containing a single genre will produce more activations with higher magnitudes than a model trained on one genre and inferred on a different genre. If the model is trained on multiple genres, some part activations occur only on a subset of songs, which contain structures those activated parts describe. Other parts, especially lower-layer parts, will activate on a majority of songs with common basic structure. In this aspect, the proposed approach can be observed as a probabilistic model for rhythmic pattern discovery and can be employed for intra- and inter-corpus analysis.

The results indicate that the model can unsupervisedly extract rhythmic patterns in a variety of conditions, without incorporating any assumptions on the specific meters or tempos. The patterns emerge as a consequence of statistical regularities in the signal, while their relative encoding makes them invariant to tempo changes. Therefore, the model is useful for rhythm analysis in a variety of musical genres and also for non-Western music with more varied rhythms. The experiment that involved large tempo changes showed the model's ability to adjust the relatively encoded structures to the tempo of the input signal and scale their activations accordingly.

The model provides the basis for several research directions which we intend to pursue in the future. On one side, we will focus on the applicability of the model to specific problems musicologists and music theorists experience in their research while using existing analytical tools. The model can be used as support for semi-automatic music analysis, and we plan to make it more suitable for individual use cases by developing user interfaces for learning and inference, as well as better visualization and representation of the learned structures. Moreover, the proposed rhythmic model could be used as a feature extractor for machine learning supported analyses and classification. Such application of the model was previously demonstrated with the symbolic version of the proposed model [26] for tune family classification. In terms of rhythm classification, the parts can be observed as features and their activations could be translated to the values of these features. By selecting a subset of layers in a learned model, the desired granularity of the features could be adjusted.

In its current state, the model discovers rhythmic patterns on a simple onset notation. To improve its performance in learning complex rhythms, we will extend the model with accent encoding in part structures. Additionally, we will extend the model with pitch information, thus merging it with the compositional hierarchical model SymCHM [25] used for pattern discovery in symbolic music. While the SymCHM discovers repeating melodic patterns in data, it ignores the rhythmic information. Ren et al. [29] analyzed the results of different approaches and features in the classification of folk songs and discovered the significance of rhythm-related features for discovery. The combined rhythmic/melodic model could potentially improve the results in melodic pattern discovery and related tasks.

Author Contributions: Conceptualization, M.M. and A.L.; methodology, M.M. and M.P.; software, M.P. and M.M.; validation, M.P. and M.M.; formal analysis, M.P.; investigation, M.M.; resources, M.M.; data curation, M.P.; writing—original draft preparation, M.P.; writing—review and editing, M.M.; visualization, M.P.; supervision, M.M. and A.L. All authors have read and agree to the published version of the manuscript.

Funding: This research received no external funding

Conflicts of Interest: The authors declare no conflict of interest. 


\section{References}

1. Brochard, R.; Abecasis, D.; Potter, D.; Ragot, R.; Drake, C. The "Ticktock" of Our Internal Clock: Direct Brain Evidence of Subjective Accents in Isochronous Sequences. Psychol. Sci. 2003, 14, 362-366. [CrossRef] [PubMed]

2. Hansen, N.C.; Sadakata, M.; Pearce, M. Nonlinear Changes in the Rhythm of European Art Music: Quantitative Support for Historical Musicology. Music Percept. Interdiscip. J. 2016, 33, 414-431. [CrossRef]

3. Toussaint, G.T. The Geometry of Musical Rhythm; Chapman and Hall/CRC: Boca Raton, FL, USA, 2016; doi:10.1201/b13751. [CrossRef]

4. Bello, J.P.; Rowe, R.; Guedes, C.; Toussaint, G. Five Perspectives on Musical Rhythm. J. New Music Res. 2015, 44, 1-2. [CrossRef]

5. Sachs, C. Rhythm and Tempo: An Introduction. Music. Q. 1952, 38, 384-398. [CrossRef]

6. Downie, J.S. The music information retrieval evaluation exchange (2005-2007): A window into music information retrieval research. Acoust. Sci. Technol. 2008, 29, 247-255. [CrossRef]

7. Dixon, S.; Gouyon, F.; Widmer, G. Towards Characterisation of Music via Rhythmic Patterns. In Proceedings of the International Conference on Music Information Retrieval (ISMIR), Barcelona, Spain, 10-14 October 2004; pp. 509-516.

8. Esparza, T.M.; Bello, J.P.; Humphrey, E.J. From Genre Classification to Rhythm Similarity: Computational and Musicological Insights. J. New Music Res. 2015, 44, 39-57. [CrossRef]

9. Böck, S.; Krebs, F.; Widmer, G. Accurate Tempo Estimation Based on Recurrent Neural Networks and Resonating Comb Filters. In Proceedings of the International Conference on Music Information Retrieval (ISMIR), Málaga, Spain, 26-30 October 2015; pp. 625-631.

10. Bock, S. Submission to the MIREX 2018: BeatTracker. In Proceedings of the MIREX 2018, Paris, France, 23-27 September 2018; pp. 1-2.

11. Bock, S.; Krebs, F. Submission to the MIREX 2018: DBNBeatTracker. In Proceedings of the MIREX 2018, Paris, France, 23-27 September 2018; pp. 1-2.

12. Krebs, F.; Böck, S.; Widmer, G. Rhythmic pattern modeling for beat and downbeat tracking in musical audio. In Proceedings of the International Conference on Music Information Retrieval (ISMIR), Curitiba, Brazil, 4-8 November 2013; pp. 1-6.

13. Huron, D. Tone and Voice: A Derivation of the Rules of Voice-Leading from Perceptual Principles. Music Percept. 2001, 19, 1-64. [CrossRef]

14. Huron, D.B. Sweet Anticipation: Music and the Psychology of Expectation; MIT Press: Cambridge, MA, USA, 2006; p. 462.

15. Abdallah, S.; Plumbley, M. Information dynamics: Patterns of expectation and surprise in the perception of music. Connect. Sci. 2009, 21, 89-117. [CrossRef]

16. Sioros, G.; Davies, M.E.P.; Guedes, C. A generative model for the characterization of musical rhythms. J. New Music Res. 2018, 47, 114-128. [CrossRef]

17. Foscarin, F.; Jacquemard, F.; Rigaux, P. Modeling and Learning Rhythm Structure. In Proceedings of the Sound \& Music Computing Conference, Málaga, Spain, 28-31 May 2019.

18. Simonetta, F.; Carnovalini, F.; Orio, N.; Rodà, A. Symbolic Music Similarity through a Graph-Based Representation. In Proceedings of the Audio Mostly 2018 on Sound in Immersion and Emotion-AM'18, Wrexham, UK, 12-14 September 2018; ACM Press: New York, NY, USA, 2018; pp. 1-7. [CrossRef]

19. Temperley, D. A Unified Probabilistic Model for Polyphonic Music Analysis. J. New Music Res. 2009, 38, 3-18. [CrossRef]

20. Temperley, D. Modeling Common-Practice Rhythm. Music Percept. Interdiscip. J. 2010, 27, 355-376. [CrossRef]

21. Holzapfel, A. Relation Between Surface Rhythm and Rhythmic Modes in Turkish Makam Music. J. New Music Res. 2015, 44, 25-38. [CrossRef]

22. London, J.; Polak, R.; Jacoby, N. Rhythm histograms and musical meter: A corpus study of Malian percussion music. Psychon. Bull. Rev. 2017, 24, 474-480. [CrossRef] [PubMed]

23. Panteli, M.; Dixon, S. On the Evaluation of Rhythmic and Melodic Descriptors for Music Similarity. In Proceedings of the International Conference on Music Information Retrieval (ISMIR), New York City, NY, USA, 7-11 August 2016; pp. 468-474. 
24. Pesek, M.; Leonardis, A.; Marolt, M. Robust Real-Time Music Transcription with a Compositional Hierarchical Model. PLoS ONE 2017, 12. [CrossRef] [PubMed]

25. Pesek, M.; Leonardis, A.; Marolt, M. SymCHM-An Unsupervised Approach for Pattern Discovery in Symbolic Music with a Compositional Hierarchical Model. Appl. Sci. 2017, 7, 1135. [CrossRef]

26. Pesek, M.; Žerovnik, M.; Leonardis, A.; Marolt, M. Modeling song similarity with unsupervised learning. In Proceedings of the Folk Music Analysis Workshop, Thessaloniki, Greece, 26-29 June 2018; pp. 1-3.

27. Pesek, M.; Leonardis, A.; Marolt, M. A compositional hierarchical model for music information retrieval. In Proceedings of the International Conference on Music Information Retrieval (ISMIR), Taipei, Taiwan, 27-31 October 2014; pp. 131-136.

28. Schluter, J.; Bock, S. Musical Onset Detection with Convolutional Neural Networks. In Proceedings of the 6th International Workshop on Machine Learning and Music, Held in Conjunction with the European Conference on Machine Learning and Principles and Practice of Knowledge Discovery in Databases, ECML/PKDD 2013, Prague, Czech Republic, 23 September 2013.

29. Ren, I.Y.; Volk, A.; Swierstra, W.; Veltkamp, R.C. Analysis by Classification: A Comparative Study of Annotated and Algorithmically Extracted Patterns in Symbolic Music Data. In Proceedings of the International Conference on Music Information Retrieval (ISMIR), Paris, France, 23-27 September 2018; pp. 539-546.

(C) 2019 by the authors. Licensee MDPI, Basel, Switzerland. This article is an open access article distributed under the terms and conditions of the Creative Commons Attribution (CC BY) license (http://creativecommons.org/licenses/by/4.0/). 